\title{
Global and local indeterminacy and optimal environmental public policies in an economy with public abatement activities
}

\author{
Rafaela Ma Pérez Sánchez \\ Departamento Fundamentos Análisis Económico I \\ Universidad Complutense \\ 28223 Madrid \\ Jesús Ruiz Andújar \\ Departamento Fundamentos Análisis Económico II \\ Universidad Complutense \\ 28223 Madrid
}

\begin{abstract}
We study the dynamic properties of an endogenous growth model with pollution in which the government can control the pollution through distorting taxes on the pollutant firms and through public abatement activities. First, we characterize the conditions for indeterminacy of equilibria when the government is benevolent and chooses its tax policy by taking into account the decentralized competitive equilibrium. Under this second best setup we show that two balanced growth paths can be found (one with a low level of pollution and the other with a high level) both of which can be locally indeterminate. Therefore, under indeterminacy, the optimal public policies do not guarantee that the economy will converge towards the steady state characterized by a low level of pollution and neither guarantee that the economy will display, along the transition, low levels of pollution. Second, we show that the central planner solution might also display indeterminacy; in particular, two Pigouvian taxes can be found.
\end{abstract}

\section{RESUMEN}

Estudiamos las propiedades dinámicas de un modelo de crecimiento endógeno con contaminación en el que el gobierno puede controlar la contaminación mediante impuestos distorsionantes sobre las empresas que contaminan y mediante actividades públicas encaminadas a reducir la contaminación. Primero, caracterizamos las condiciones para que se produzca indeterminación de equilibrios cuando el gobierno es benevolente y elige su política fiscal teniendo en cuenta el equilibrio descentralizado. Demostramos que, en este contexto de second-best, puede encontrarse indeterminación global (puede haber dos sendas de crecimiento equilibrado) y local. Por tanto, bajo indeterminación, la politica económica óptima no garantiza que la economía convergerá hacia el estado estacionario caracterizado por un nivel de contaminación bajo y tampoco garantiza que, durante la transición, la economía mostrará bajos niveles de emisiones. Segundo, demostramos que la solución del planificador también puede estar indeterminada, en particular, pueden existir dos impuestos Pigouvianos.

The authors would like to thank professors A. Novales, S. Smulders and the participants at the 1st. Atlantic Workshop on Energy and Environmental Economics for helpful comments and financial support from Fundación CentrA, Fundación Ramón Areces and from the Spanish Ministry of Education through grant BEC2003-03965. Address for correspondence: Jesús Ruiz Andújar. Dpto. Fundamentos Análisis Económico II. Facultad CCEE. UCM. 28223 Campus de Somosaguas. Madrid. Tfno. 34913942352. E-mail: jruizand@ccee.ucm.es 


\section{Introduction}

Nowadays there exist legal standards on the socially acceptable ambient concentration level of pollutant. However, a producer has an incentive to shirk on pollution control. Since a producer's profits come from a market price that does not reflect society's preferences for environmental protection, the producer has no economic incentive to supply the level of pollution control society wants. If the market is not sending the correct signal to the producer about the socially optimal level of pollution control, a regulator can implement economic incentives that increase the cost of shirking on pollution control. For example, the government could tax the polluting productive activities. Nevertheless, a coordination failure between private and government sectors could arise because the choice of tax policy affects private agents' decisions and, at the same time, government expenditures could yield external benefits to private agents. As a consequence, the environmental policy implemented by government could not yield the effects on pollution level that society wants. From a theoretical point of view, the existence of coordination failure can be explained through the indeterminacy of equilibria issue.

This paper studies the dynamic properties of a general equilibrium model of endogenous growth with environmental externalities. In particular, we study the conditions for global and local indeterminacy, and their implications for the governmental control of pollution.

General equilibrium models that display indeterminacy have been focus of attention in recent years. Global indeterminacy implies multiple balanced growth paths (BGP) along which the economy can persistently grow in the long run. On the other hand, local indeterminacy implies that there will be multiple paths converging to a given steady state. Hence, indeterminacy guarantees existence of a continuum of sunspot stationary equilibria, i.e., stochastic rational expectations equilibria determined by perturbations unrelated to the uncertainty in economic fundamentals.

Characteristics that produce indeterminacy of equilibria in one- or multi-sector real business cycle models or in endogenous growth models have been widely studied. Initially, it was shown that when these models are extended to include either productivity externalities or some market imperfection, indeterminacy can arise if social returns to scale in production are sufficiently high so that the labor demand curve has a slope which is not only positive, but also greater than that of the labor supply curve (see Benhabib and Farmer (1994), and Farmer and Guo (1994), for one-sector models). These models have been widely criticized because to produce multiple equilibria they require larger returns to scale than observed in actual data [see Aiyagari (1995)]. However, Fernández, Novales and Ruiz (2003) obtain multiple equilibria under constant returns to scale and endogenous government expenditures included in the utility function.

More recent work has described two situations in which increasing returns needed to produce indeterminacy are lower than initially thought, sometimes allowing for negatively sloped labor demand curves: i) two sector economies with externalities in one sector (Benhabib and Farmer (1996), Perli (1998), Benhabib and Nishimura (1998)); ii) one sector models with non-standard characteristics, such as non-separable preferences (Bennett and Farmer (2000)), or capacity utilization affecting production (Wen (1998)). These models are able to produce indeterminacy of equilibria with relatively low increasing returns to 
scale, but the elasticity of intertemporal substitution needed is often too large, relative to values considered in the real business cycle literature (Weder (1998) and Bennett and Farmer (2000), among others). This will generally lead to rather volatile consumption paths, against the observation in most actual economies. The same criticism applies to existing endogenous growth models producing indeterminacy (see Benhabib and Perli (1994), and Xie (1994)).

All these previous papers study indeterminacy for the decentralized solution. However, we focus our interest in the indeterminacy conditions for the Ramsey-type fiscal policy problem. In this analysis, we follow the methodology used by Park and Philippopoulos (2004) who study indeterminacy in an economy without pollution very different to ours, in which capital taxes are used to finance public production and consumption services.

In our paper, the economy includes the following features: i) pollution is a side-effect of the productive activity and enters negatively in the utility function of agents (Smulders and Gradus (1996), Ligthart and van der Ploeg (1994), or Bovenberg and de Mooij (1997), ${ }^{1}$ are some examples), who value negatively the effects of pollution, for example, the possibility of a climate change for global warming, its damage on human health or pollution of rivers and lakes; ii) the government provides two types of services, first, services that increase the labor efficiency in the productive sector and, second, services that improve the environmental quality ('abatement activities'); iii) public expenditures are financed by a proportional tax charged on firms' revenues (firms finance the abatement activities because they recieve the benefits from the labor productivity improvement and because they also perform the polluting productive activity).

Under this framework, the government chooses in every moment of time the optimal composition of public spending, together with the optimal tax rate, taking into acount the decentralized competitive equilibrium. We study how second-best economic policy influences the dynamics of growth, a task that has not been previously undertaken in environmental economics literature. We show that when we include pollution entering in the utility function together with the abatement public activities, the introduction of endogenously chosen economic policy generates an expectations coordination problem: Although the decentralized solution is determinate (globally and locally), we find that the 'second-best' solution can be globally and locally indeterminate, that is, there can be two steady states (global indeterminacy) both of which can be locally indeterminate (that is, there exist multiple converging paths to each state).

We show that the two long-run equilibria are optimal and that they have different properties. One of them has a large pollution level and a large growth rate; the other has a low level for both pollution and growth rate. Furthermore, one of then could display local indeterminacy whilst the other could be locally determinate.

We also show that the environmental externality in the utility function is a key factor to obtain global indeterminacy. On the contrary, the other type of externality in our economy is not crucial to achieve the indeterminacy result, that is, if the public services do not provide any production externalities, still is possible to obtain a global indeterminacy result.

\footnotetext{
${ }^{1}$ They include environmental quality as a positive externality in the utility function. However, environmental quality is inversely related to pollution, leading to the same welfare function employed in our model.
} 
The economic implication behind global indeterminacy is that two economies with the same fundamentals could choose different optimal policies (taxes and spending composition) that would yield different optimal paths of pollution. This result is able to explain the lack of convergence in the pollution levels of countries with similar fundamentals.

On the other hand, local indeterminacy implies that there are multiple converging paths towards a given balanced growth path (BGP). The main implication of this result for environmental policy is the following: under indeterminacy situations, public policies are insufficient to drive the economy towards low pollution levels during the transition towards the long-run equilibrium. The agents' decisions (private and government sectors), independent of initial conditions or other fundamentals, will place the economy in a particular optimal converging path and it could not coincide with the path corresponding to the lowest pollution levels.

In our model, local indeterminacy is able to explain why two countries with similar fundamentals (preferences, technology and initial capital stock) might display along the time different relationships between development degree and environmental quality (or pollution). This relationship is known as the Environmental Kuznets Curve (EKC) and the existence of local indeterminacy could be a possible explanation to the difficulty to find a clear empirical regularity supporting the EKC hypothesis (see de Bruyn and Heintz, 1999).

Finally, we compare the second-best tax rate and the pollution path under the Ramsey solution with the first-best tax (Pigouvian tax) and the efficient pollution path, under the planner solution. By comparing the first-order conditions of the decentralized solution with the corresponding first-order conditions of the planner solution, we are able to determine the first-best tax rate and spending composition. Such pigouvian tax rate and spending composition let the economy reach the efficient path as a competitive equilibrium, that is, they internalize the externalities. We show that there are structural parameter sets for which the pigouvian tax rate does not exist, that is, it is not possible to find a tax rate so that the competitive equilibrium replicate the efficient path. However, there are also structural parameter sets for which there exist two pigouvian tax rates. In these cases, the environmental quality is worse under the planner solution than under Ramsey problem since pollution is growing at a larger rate, the share of public spending devoted to abatement is lower and the first-best tax rate is also lower.

The model is described in section 2 . In section 3 we characterize the second-best policy and study the conditions for global and local indeterminacy. In section 4 the efficient balanced growth path is studied and the pigouvian tax is computed. The paper closes with some remarks in section 5 .

\section{The economy}

We consider a closed economy that displays endogenous growth with constant population normalized to one. In this section we set up the decentralized equilibrium. The private sector consists of a representative household and a representative firm, both acting competitively. Pollution appears as a production externality and has a negative effect on the household utility. The goverment sector taxes the representative firm (who pollutes 
pay) and uses the revenues to finance two types of government spending: i) abatement activities that improve environmental quality and ii) spending that increases the labor efficiency in the productive sector.

\subsection{The household}

The representative household maximizes intertemporal utility:

$$
\int_{0}^{\infty} e^{-\rho t}[\ln c-\eta \ln P] d t
$$

where $c$ is consumption per capita, $P$ is the flow of pollution, the parameter $\rho>0$ is the rate of time preference and $\eta>0$ is the weight of pollution in utility. To simplify notation, time dependence of all variables is suppressed.

The household consumes and accumulates capital, and recieves income from labor (which offers in an inelastic manner) and from capital renting to the firms. Thus the household constraint is:

$$
c+\dot{k}=r k+w
$$

where $r$ is the market return to capital and $w$ the wage rate. A dot over a variable denotes time derivative and the initial stock $k_{0}>0$ is given.

The representative household is competitive, and hence, takes prices and pollution as given. The control variables are $c$ and $k$, so that the first-order conditions are the standard Euler condition

$$
\frac{\dot{c}}{c}=r-\rho,
$$

apart from the budget constraint and the transversality condition

$$
\lim _{t \rightarrow \infty} e^{-\rho t} \lambda k=0 .
$$

\subsection{The firm}

The competitive representative firm takes prices and public variables (taxes and government expenditures) as exogenously determined. It maximizes profits, given by:

$$
\pi=(1-\tau) Y-r K-w L
$$

subject to the technology constraint:

$$
Y=A(g L)^{1-\alpha} K^{\alpha}, \text { with } 0<\alpha<1,
$$

where $\tau$ is the tax rate on firm's revenues, $Y, g, K$ and $L$ are, respectively, the aggregate levels for output, the flow of public investment (government spending that increases private labor productivity), and private capital and labor. 
Technology can also be expressed in per capita terms or per labor units:

$$
y=A g^{1-\alpha} k^{\alpha}
$$

and hence, it is assumed that public productive services are not subject to congestion.

The first order condition for capital and labor are as usual:

$$
\begin{aligned}
r & =(1-\tau) \alpha \frac{y}{k}, \\
w & =(1-\tau)(1-\alpha) y .
\end{aligned}
$$

\subsection{Government sector}

The government budget is balanced in every period of time:

$$
\tau y=g+z,
$$

where $\tau y=\tau A g^{1-\alpha} k^{\alpha}$ are total tax revenues and $g+z$ are total government spending, $z$ being the expenditures allocated to increasing the environmental quality, that is, the flow of abatement activities.

The allocation of total government spending between investment and abatement is made by following the rules:

$$
\begin{aligned}
& g=(1-\phi) \tau A g^{1-\alpha} k^{\alpha} \\
& z=\phi \tau A g^{1-\alpha} k^{\alpha}
\end{aligned}
$$

where $\phi$ is the share of public spending devoted to abatement activities. We will refer to $\phi$ as public spending composition.

From (7) and (8):

$$
\begin{aligned}
\frac{g}{k} & =[A \tau(1-\phi)]^{1 / \alpha} \\
z & =\frac{\phi}{(1-\phi)} g
\end{aligned}
$$

\subsection{Pollution equation}

We adopt the pollution function proposed by Smulders and Gradus (1996), ${ }^{2}$ that is, pollution flow depends positively on the capital stock use and negatively on the abatement activities, i.e. $P=f(K, Z)=f(k, z),{ }^{3}$ where $f_{k}>0$ and $f_{z}<0 .{ }^{4}$ Hence, by using (9) and (10) in this definition:

$$
P=\frac{k^{\chi_{1}}}{z^{\chi_{2}}}=\frac{k^{\chi_{1}}}{\left[\frac{\phi}{(1-\phi)} g\right]^{\chi_{2}}}=\frac{k^{\chi_{1}-\chi_{2}}}{\left[\phi^{\alpha}(1-\phi)^{(1-\alpha)} \tau A\right]^{\chi_{2} / \alpha}}
$$

\footnotetext{
${ }^{2}$ Mohtadi (1996) adopt a similar function for the level of the environmental quality, where the environmental quality is inversely related to pollution flow.

${ }^{3}$ Note that we assume constant population normalized to one. Hence, $K=k, Z=z$.

${ }^{4}$ We define $f_{x}=\frac{\partial f}{\partial x}$, for $x=k, z$.
} 


\subsection{Decentralized Competitive Equilibrium}

Definition 1 A competitive equilibrium for this economy is a set of allocations $\{c, k, l\}_{t=0}^{\infty}$ and a price system $\{w, r\}_{t=0}^{\infty}$ such that given a price system and a fiscal policy $\{\tau, \phi\}_{t=0}^{\infty}$ : i) $\{c, k\}_{t=0}^{\infty}$ maximizes household's utility (1), subject to (2), and taking the pollution level $\{P\}_{t=0}^{\infty}$ and $\left\{k_{0}\right\}$ as given; ii) $\{k, l\}_{t=0}^{\infty}$ satisfies the firms profit maximization conditions, and iii) all markets clear, that is, $\{c, k, g, z\}_{t=0}^{\infty}$ satisfies the aggregate resources constraint (12) below and the government budget constraint (6) and, in the labor market, $l=1$ :

$$
c+k=(1-\tau) A g^{1-\alpha} k^{\alpha} .
$$

By using (3), (4) and (9):

$$
\frac{\dot{c}}{c}=\Psi(\tau, \phi)-\rho
$$

where

$$
\Psi(\tau, \phi)=(1-\tau) \alpha A^{1 / \alpha}[\tau(1-\phi)]^{(1-\alpha) / \alpha}
$$

From (2), (4), (5) and (9):

$$
\frac{\dot{k}}{k}=\frac{\Psi(\tau, \phi)}{\alpha}-\frac{c}{k}
$$

Note that, from (13), the long-run growth rate is positive if and only if $\Psi(\tau, \phi)>\rho$. Hence, we will only consider balanced growth paths in which the time paths for $\tau, \phi$ guarantee that this condition holds.

The decentralized equilibrium is globally and locally determinate. In the end, this model is quite similar to Barro's (1990) model, and it is well known that Barro's model is locally and globally determinate.

\section{Second-best policy}

The government chooses economic policy $\{\tau, \phi\}$ to maximize the utility of the representative household, subject to the competitive equilibrium conditions. That is:

$$
\begin{aligned}
& \underset{\{c, k, P, \tau, \phi\}}{\operatorname{Max}} \int_{0}^{\infty} e^{-\rho t}[\ln c-\eta \ln P] d t \\
\text { s.t. } & : \\
\dot{c}= & c[\Psi(\tau, \phi)-\rho] \\
\dot{k}= & \frac{\Psi(\tau, \phi)}{\alpha} k-c \\
P= & k^{\chi_{1}-\chi_{2}}\left[\phi^{\alpha}(1-\phi)^{(1-\alpha)} \tau A\right]^{-\chi_{2} / \alpha}
\end{aligned}
$$

We refer to this optimal control problem as the Ramsey problem and to the associated allocations and policies as the Ramsey allocations and the Ramsey plan. We assume that 
the government has some commitment power to bind itself to implement, at any future date, the plan it has announced in the first period.

The current-value Hamiltonean is:

$$
\begin{aligned}
H \equiv & e^{-\rho t}\left[\ln c-\eta\left(\chi_{1}-\chi_{2}\right) \ln k+\ldots\right. \\
& \left.+\eta \chi_{2}\left(\ln \phi+\frac{1-\alpha}{\alpha} \ln (1-\phi)+\frac{1}{\alpha} \ln (\tau A)\right)\right]+\ldots \\
& +e^{-\rho t} \mu_{1}[c(\Psi(\tau, \phi)-\rho)]+e^{-\rho t} \mu_{2}\left[\frac{\Psi(\tau, \phi)}{\alpha} k-c\right]
\end{aligned}
$$

where $\mu_{1}$ and $\mu_{2}$ are the dynamic multipliers associated to (16) and (17), respectively.

The necessary conditions with respect to $\left\{c, k, \mu_{1}, \mu_{2}, \tau, \phi\right\}$ are given by:

$$
\begin{aligned}
-\frac{\partial H}{\partial c} & =\dot{\mu}_{1}-\rho \mu_{1} \rightarrow \dot{\mu}_{1}=-\frac{1}{c}+\mu_{2}-\mu_{1}[\Psi-\rho]+\rho \mu_{1}, \\
-\frac{\partial H}{\partial k} & =\dot{\mu}_{2}-\rho \mu_{2} \rightarrow \dot{\mu}_{2}=\mu_{2}\left[\rho-\frac{\Psi}{\alpha}\right]+\eta\left(\chi_{1}-\chi_{2}\right) \frac{1}{k}, \\
\frac{\partial H}{\partial \mu_{1}} & =0 \rightarrow \dot{c}=c[\Psi-\rho], \\
\frac{\partial H}{\partial \mu_{2}} & =0 \rightarrow \dot{k}=\frac{\Psi}{\alpha} k-c, \\
\frac{\partial H}{\partial \tau} & =0 \rightarrow 0=\frac{\eta \chi_{2}}{\alpha} \frac{1}{\tau}+\mu_{1} c \Psi_{\tau}+\mu_{2} \frac{k}{\alpha} \Psi_{\tau}, \\
\frac{\partial H}{\partial \phi} & =0 \rightarrow 0=\eta \chi_{2} \frac{\alpha-\phi}{\alpha \phi(1-\phi)}+\mu_{1} c \Psi_{\phi}+\mu_{2} \frac{k}{\alpha} \Psi_{\phi} \\
& \rightarrow 0=\eta \chi_{2} \frac{\alpha-\phi}{\phi}+\mu_{1} c(\alpha-1) \Psi+\mu_{2} \frac{(\alpha-1) k}{\alpha} \Psi,
\end{aligned}
$$

where $\Psi=\Psi(\tau, \phi), \Psi_{\tau}=\frac{\partial \Psi}{\partial \tau}=\frac{1-\tau-\alpha}{\tau \alpha} \Psi, \Psi_{\phi}=\frac{\partial \Psi}{\partial \phi}=\frac{(\alpha-1)}{\alpha} \frac{\Psi}{(1-\phi)}$.

Proposition 1 The optimal ratio of abatement to total government spending, $\phi$, falls between 0 and $\alpha^{2}$, and the optimal tax rate, $\tau$, falls between $(1-\alpha)$ and 1 , for all $t$.

Proof. By using (23) and (24), we obtain that

$$
\phi=\alpha\left(1-\frac{1-\alpha}{\tau}\right)
$$

Hence, it is obvious that $\phi$ is monotonically increasing and concave in $\tau$. Because $\phi$ and $\tau$ must be defined in the interval $(0,1)$, and given $(25)$, it is readily obtained that the range for the function $(25)$ is given by $[1-\alpha, 1)$ and the domain is given by $\left[0, \alpha^{2}\right)$.

Corollary 2 The variation rate of optimal $\phi$ is monotonically increasing in the variation rate of optimal $\tau$. 
Proof. From (25), we obtain the instantenous change for optimal $\phi$

$$
\dot{\phi}=\frac{\alpha(1-\alpha)}{\tau} \frac{\dot{\tau}}{\tau}
$$

and dividing this expression by $\phi$, we obtain

$$
\frac{\dot{\phi}}{\phi}=\frac{(1-\alpha)}{\tau-(1-\alpha)} \frac{\dot{\tau}}{\tau}
$$

Given Proposition (1), $\frac{(1-\alpha)}{\tau-(1-\alpha)}>0$, and hence, $(27)$ is monotonically increasing in $\frac{\dot{\tau}}{\tau}$.

The system (19) - (24) consists of six equations and six variables. We reduce its dimensionality to simplify computation. The new variables vector will be $\left\{x_{1}, x_{2}, \tau\right\}$, where $x_{1}$ and $x_{2}$ are two auxiliary variables defined as:

$$
\begin{aligned}
& x_{1} \equiv \frac{c}{k}, \\
& x_{2} \equiv \mu_{2} c,
\end{aligned}
$$

so that both $x_{1}$ and $x_{2}$ do not grow in the steady state. This implies that $c$ and $k$ grow at the same rate, denoted by $\gamma$.

Differentiating (28), (29), and (23) with respect to time and using (19) to (24), we obtain after some algebra:

$$
\begin{gathered}
\dot{x}_{1}=\left[\tilde{\Psi}\left(1-\frac{1}{\alpha}\right)-\rho+x_{1}\right] x_{1}, \\
\dot{x}_{2}=\tilde{\Psi}\left(1-\frac{1}{\alpha}\right) x_{2}+\eta\left(\chi_{1}-\chi_{2}\right) x_{1}, \\
\dot{\tau}=\frac{1}{\eta \chi_{2}} \Theta \Omega,
\end{gathered}
$$

where: ${ }^{5}$

$$
\begin{gathered}
\tilde{\Psi}=\tilde{\Psi}(\tau)=(1-\tau) \alpha A^{1 / \alpha}[(1-\alpha)(\tau+\alpha)]^{(1-\alpha) / \alpha}, \\
\Theta=\Theta(\tau)=\frac{\alpha(1-\tau)(\tau+\alpha)(1-\tau-\alpha)^{2} \tilde{\Psi}}{(1-\tau-\alpha)\left[1-\tau-\alpha-\alpha^{2}\right]-\alpha(1-\tau)(\tau+\alpha)}, \\
\Omega=\Omega\left(x_{2}, \tau\right)=\frac{\eta\left(\chi_{1}-\chi_{2}\right)}{\alpha}-1-\frac{x_{2}(1-\alpha)}{\alpha}-\frac{\rho \eta \chi_{2}}{(1-\tau-\alpha) \tilde{\Psi}} .
\end{gathered}
$$

Now, the steady state and the transitional dynamic properties of the Ramsey problem can be studied from the three-dimensional system (30)-(32) in $\left\{x_{1}, x_{2}, \tau\right\}$.

${ }^{5}$ Note that by using (25) in (14), we obtain (33). 


\subsection{Steady state and global indeterminacy}

This section focuses on the steady state or balanced growth path (BGP). An interior steady state is a vector $\left(\hat{x}_{1}, \hat{x}_{2}, \hat{\tau}\right)$ satisfying the equations for the Ramsey problem (in particular, the equations (30)-(32)), such that if it is ever reached, the system will stay at that point forever $\left(\dot{x}_{1}=\dot{x}_{2}=\dot{\tau}=0\right)$. Defining $\hat{\Psi}=\tilde{\Psi}(\hat{\tau})$, the steady state is given by

$$
\begin{gathered}
\hat{x}_{1}=\rho+\frac{(1-\alpha) \hat{\Psi}}{\alpha}, \\
\hat{x}_{2}=\eta\left(\chi_{1}-\chi_{2}\right)\left[1+\frac{\rho \alpha}{(1-\alpha) \hat{\Psi}}\right],
\end{gathered}
$$

and $\hat{\tau}$ is obtained from the implicit equation:

$$
\hat{\Psi}=\frac{\rho \eta}{1-\eta\left(\chi_{1}-\chi_{2}\right)}\left[\chi_{2} \frac{\hat{\tau}+\alpha}{(\hat{\tau}+\alpha)-1}-\chi_{1}\right] .
$$

The first stage is to find the solution for (38) in the long-run tax rate, $\hat{\tau}$. Once it has been obtained, (36) can give the consumption to capital ratio in the steady state $\left(\hat{x}_{1}\right)$ and (37) determines the long-run value for the auxiliary variable $\hat{x}_{2}$.

Proposition 3 A necessary condition for the consumption long-run growth rate to be positive is that

$$
\chi_{2} \frac{\hat{\tau}+\alpha}{(\hat{\tau}+\alpha)-1}>\chi_{1}, \text { together with } 1>\eta\left(\chi_{1}-\chi_{2}\right)
$$

or

$$
\chi_{2} \frac{\hat{\tau}+\alpha}{(\hat{\tau}+\alpha)-1}<\chi_{1} \text {, together with } 1<\eta\left(\chi_{1}-\chi_{2}\right)
$$

Proof. From (21), the consumption growth rate is positive in equilibrium if and only if $\hat{\Psi}>\rho$. Hence, a necessary condition for positive growth is $\hat{\Psi}>0$. As long as Proposition (1) implies that $\hat{\tau} \geq 1-\alpha$, and hence, $\chi_{2} \frac{\hat{\tau}+\alpha}{(\hat{\tau}+\alpha)-1}>0$, it is obvious from (38) that the condition for $\hat{\Psi}>0$ is characterized by (39) or (40).

Proposition 4 The second best consumption long-run growth rate is positive if and only if $\eta \chi_{2}>\hat{\tau}+\alpha-1$.

Proof. From (21) the consumption growth rate is positive in equilibrium if and only if $\hat{\Psi}>\rho$. From (38), $\hat{\Psi}>\rho$ if and only if $\frac{\eta}{1-\eta\left(\chi_{1}-\chi_{2}\right)}\left[\chi_{2} \frac{\hat{\tau}+\alpha}{(\hat{\tau}+\alpha)-1}-\chi_{1}\right]>1$. After some algebra, the condition $\eta \chi_{2}>\hat{\tau}+\alpha-1$ can be obtained.

In order to study the conditions for global indeterminacy, first, we characterize the shape of the left-hand side of (38), denoted by

$$
\operatorname{LHS}(\hat{\tau})=\hat{\Psi}=(1-\hat{\tau}) \alpha A^{1 / \alpha}[(1-\alpha)(\hat{\tau}+\alpha)]^{(1-\alpha) / \alpha},
$$


and the shape of the right-hand side of (38), denoted by

$$
R H S(\hat{\tau})=\frac{\rho \eta}{1-\eta\left(\chi_{1}-\chi_{2}\right)}\left[\chi_{2} \frac{\hat{\tau}+\alpha}{(\hat{\tau}+\alpha)-1}-\chi_{1}\right]
$$

Proposition 5 The function $L H S(\hat{\tau})$ is monotonically decreasing and concave and its domain is given by $\left[0, \alpha^{2} A^{1 / \alpha}(1-\alpha)^{\frac{1-\alpha}{\alpha}}\right], \forall \hat{\tau} \in[1-\alpha, 1)$.

Proof. See Appendix.

Proposition 6 If $1<\eta\left(\chi_{1}-\chi_{2}\right)$ and $\chi_{2} \frac{1+\alpha}{\alpha}<(>) \chi_{1}$, then the function $R H S(\hat{\tau})$ is monotonically increasing, concave, and its domain is $(-\infty, \kappa)$, with $\kappa>(<) 0$.

Proof. See Appendix.

Proposition 7 If $1>\eta\left(\chi_{1}-\chi_{2}\right)$ and $\chi_{2} \frac{1+\alpha}{\alpha}<(>) \chi_{1}$, then the function $R H S(\hat{\tau})$ is monotonically decreasing and convex and its domain is $(\kappa, \infty)$, with $\kappa<(>) 0$.

Proof. See Appendix.

Let $\hat{\gamma}$ and $\hat{\phi}$ be the optimal long-run levels for the growth rate and the spending composition, respectively.

Now, the conditions for global indeterminacy can be characterized. From the propositions above, four types of situations can be obtained:

i) If $R H S(\hat{\tau})$ is monotonically increasing, concave, and its domain is $(-\infty, \kappa)$, with $\kappa>0$, then $R H S(\hat{\tau})>0$ for $\hat{\tau} \rightarrow 1^{-}$. In this situation, see figure 1a, there exists one single solution for $\hat{\tau}$, which verifies the necessary condition for positive growth rate.

ii) If $R H S(\hat{\tau})$ is monotonically increasing, concave, and its domain is $(-\infty, \kappa)$, with $\kappa<0$, then $R H S(\hat{\tau})<0$ for $\hat{\tau} \rightarrow 1^{-}$. In this case, there is no solution (see figure $1 \mathrm{~b}$ ).

iii) If $R H S(\hat{\tau})$ is monotonically decreasing and convex and its domain is $(\kappa, \infty)$, with $\kappa<0$, then $R H S(\hat{\tau})<0$ for $\hat{\tau} \rightarrow 1^{-}$. Hence, there is one single solution for $\hat{\tau}$ (see figure 2 ). In this case, the fulfilment of the necessary condition for positive long-run growth rate (Proposition 2) under the optimal tax rate must be checked, because it does not hold for $\hat{\tau} \rightarrow 1^{-}$.

iv) If $R H S(\hat{\tau})$ is monotonically decreasing and convex and its domain is $(\kappa, \infty)$, with $\kappa>0$, then $R H S(\hat{\tau})>0, \forall \hat{\tau} \in[1-\alpha, 1)$. Then there are three possibilities: First, $L H S(\hat{\tau})$ lies below $R H S(\hat{\tau}), \forall \hat{\tau} \in[1-\alpha, 1$ ); in this case there is no solution (see figure 3a). Second, $\operatorname{LHS}(\hat{\tau})$ is tangent to $R H S(\hat{\tau})$; in this case, there is one single solution (see figure $3 \mathrm{~b}$ ). Third, $L H S(\hat{\tau})$ lies above $R H S(\hat{\tau})$ as shown in figure 3c; in this case, there are two solutions. This last situation guarantees the existence of global indeterminacy, that is, there exist two long-run optimal tax rates $\hat{\tau}_{1}$ and $\hat{\tau}_{2}$ such that $(1-\alpha)<\hat{\tau}_{1}<\hat{\tau}_{2}<1$. Hence, there are two Ramsey allocations corresponding to two Ramsey plan. From (25) and (13), the optimal $\left(\hat{\phi}_{1}, \hat{\gamma}_{1}\right)$ and $\left(\hat{\phi}_{2}, \hat{\gamma}_{2}\right)$ can be obtained, verifying that $0<\hat{\phi}_{1}<\hat{\phi}_{2}<\alpha^{2}$ and $\hat{\gamma}_{1}>\hat{\gamma}_{2} \cdot{ }^{6}$

\footnotetext{
${ }^{6}$ Note that in this case $\hat{\tau}$ verifies the necessary condition for positive growth rate (Proposition 2).
} 
On the other hand, if $\chi_{1}>\chi_{2}$ the pollution growth rate will be positive and for $\hat{\tau}_{1}$ will be larger than for $\hat{\tau}_{2}$. Furthermore, the share of public spending devoted to abatement is lower the lower the tax rate, and hence, the pollution level will also be larger for $\hat{\tau}_{1}$ ceteris paribus. If $\chi_{1}<\chi_{2}$, the pollution growth rate is negative, and hence, pollution will fall at a faster rate the lower $\hat{\tau}$. However, the abatement activities are lower for $\hat{\tau}_{1}$ so that the pollution path for $\hat{\tau}_{1}$ lies above the pollution path for $\hat{\tau}_{2}$ only during the first periods.

The economic implication behind global indeterminacy is that two economies with the same fundamentals could choose different optimal policies (taxes and spending composition) that would yield different optimal paths of pollution. This result is able to explain the lack of convergence in the pollution levels of countries with similar fundamentals.

It is easy to provide several numerical examples of a unique interior BGP or two interior BGPs. The first example corresponds to a unique interior BGP that can be obtained for the following set of structural parameters: $\alpha=0.7, A=1, \rho=-\log (0.95), \eta=4$, $\chi_{1}=0.8, \chi_{2}=0.1$. The optimal tax rate corresponding to this framework is $\hat{\tau}=0.88$, the optimal spending composition is $\hat{\phi}=0.46$ and the optimal growth rate is $\hat{\gamma}=0.009$. It is easy to check that with these parameter values the economy is placed in the situation displayed in figure 1a.

The second example yields a unique interior BGP corresponding to the situation in figure $3 \mathrm{~b}$. The set of structural parameters is given by: $\alpha=0.7, A=1, \rho=-\log (0.95)$, $\eta=3.310725, \chi_{1}=0.4, \chi_{2}=0.2$. The optimal tax rate is $\hat{\tau}=0.768$, the optimal spending composition is $\hat{\phi}=0.427$ and the optimal growth rate is $\hat{\gamma}=0.063$.

The third example leads to two interior BGP like the situation displayed in figure 3c. The set of parameters is: $\alpha=0.7, A=0.7, \rho=-\log (0.95), \eta=1, \chi_{1}=0.7, \chi_{2}=0.6$. For the first BGP, the optimal tax rate is $\hat{\tau}_{1}=0.58$, the optimal spending composition is $\hat{\phi}_{1}=0.34$ and the optimal growth rate is $\hat{\gamma}_{1}=0.067$. The second BGP is defined by $\hat{\tau}_{2}=0.78, \hat{\phi}_{2}=0.43, \hat{\gamma}_{2}=0.0145$.

\subsection{Source of indeterminacy}

In this model, the environmental externality in the utility function is key to obtain the result of global indeterminacy. We show that indeterminacy is not present in the case of $\eta=0$, that is, pollution does not affect the agent's welfare: if $\eta=0$, equation (23) becomes

$$
\left[\mu_{1} c+\mu_{2} \frac{k}{\alpha}\right] \quad \Psi_{\tau}=0 .
$$

The term in brackets is never zero, ${ }^{7}$ so the expression above only holds if $\Psi_{\tau}=0$. This condition is only guaranteed for $\tau=1-\alpha$. The simplification achieved by imposing $\eta=0$, leads to Barro (1990) model, and because of that, the optimal tax rate is the same obtained by him. In this simplified economy, the optimal percentage of public spending devoted to abatement, $\phi$, is zero, since there is no incentive to reduce the pollution level.

\footnotetext{
${ }^{7}$ Differentiation of the term in brackets with respect to time yields an expression that is not compatible with the other first order conditions (19)-(22).
} 
On the contrary, the other type of externality in our benchmark economy is not crucial to achieve the indeterminacy result. If the public services do not provide any production externalities $(\alpha=1)$, still is possible to obtain a global indeterminacy result. If $\alpha=1$, the optimal $\phi$ is equal to 1 , that is, there is no incentives to allocate public spending to public services in production. In this case, the equation (23) becomes

$$
\frac{\eta \chi_{2}}{A \tau(1-\tau)}=\left[\mu_{1} c+\mu_{2} \frac{k}{\alpha}\right]
$$

Differentiating with respect to time the expression (42) and using (19)-(22), we obtain

$$
\frac{\dot{\tau}}{\tau} \frac{(1-2 \tau) \eta \chi_{2}}{A \tau(1-\tau)}+(1-\tau)\left[-1+\eta\left(\chi_{1}-\chi_{2}\right)+\frac{\rho \eta \chi_{2}}{A \tau(1-\tau)}\right]=0 .
$$

From $(43)$, in the steady state $(\dot{\tau}=0)$ the optimal tax rate $(\hat{\tau})$ must verify

$$
\left[1-\eta\left(\chi_{1}-\chi_{2}\right)\right] \hat{\tau}=\frac{\rho \eta \chi_{2}}{A(1-\hat{\tau})}, \forall \hat{\tau} \in(0,1)
$$

It is readily obtained that the right-hand side of equation (44) is positive, increasing, convex, with range $(0,1)$ and domain $\left(\frac{\rho \eta \chi_{2}}{A}, \infty\right)$. On the other hand, the left-hand side is a ray with slope $1-\eta\left(\chi_{1}-\chi_{2}\right)$. If $1<\eta\left(\chi_{1}-\chi_{2}\right)$, there is no solution for $\hat{\tau} \in(0,1)$ verifying (44). But if $1>\eta\left(\chi_{1}-\chi_{2}\right)$, then we can find no solution, one or two solutions for $\hat{\tau}$. This last case corresponds to a global indeterminacy situation.

\subsection{Transitional dynamics and local indeterminacy}

Linearizing the dynamic system (30)-(32) around the BGP in (36)-(38), allows to obtain the following linear system:

$$
\left[\begin{array}{c}
\dot{x}_{1} \\
\dot{x}_{2} \\
\dot{\tau}
\end{array}\right]=\left[\begin{array}{ccc}
\hat{x}_{1} & 0 & \hat{x}_{1} \hat{\Psi}_{\tau}\left(1-\frac{1}{\alpha}\right) \\
\eta\left(\chi_{1}-\chi_{2}\right) & \hat{\Psi}\left(1-\frac{1}{\alpha}\right) & \hat{x}_{2} \hat{\Psi}_{\tau}\left(1-\frac{1}{\alpha}\right) \\
0 & \hat{\Xi} & \rho
\end{array}\right]\left[\begin{array}{c}
x_{1}-\hat{x}_{1} \\
x_{2}-\hat{x}_{2} \\
\tau-\hat{\tau}
\end{array}\right]
$$

where: $\hat{\Psi}_{\tau}=\frac{\partial \tilde{\Psi}(\hat{\tau})}{\partial \tau}=\frac{1-\hat{\tau}-\alpha-\alpha^{2}}{\alpha(1-\hat{\tau})(\hat{\tau}+\alpha)} \hat{\Psi}$, and $\hat{\Xi}=-\frac{\hat{\Psi}_{\tau} \alpha(1-\alpha)(1-\hat{\tau})(\hat{\tau}+\alpha)(1-\hat{\tau}-\alpha)^{2}}{\alpha \eta \chi_{2}\left[(1-\hat{\tau}-\alpha)\left(1-\hat{\tau}-\alpha-\alpha^{2}\right)-\alpha(1-\hat{\tau})(\hat{\tau}+\alpha)\right]}$.

Let $\Gamma$ be the Jacobian matrix in (45) and the characteristic equation of $\Gamma$ is:

$$
w^{3}-\operatorname{tr}(\Gamma) w^{2}+\Omega(\Gamma) w-\operatorname{det}(\Gamma)=0,
$$

where:

$$
\begin{aligned}
\operatorname{tr}(\Gamma) & =2 \rho \\
\operatorname{det}(\Gamma) & =\left(1-\frac{1}{\alpha}\right) \rho \hat{x}_{1} \hat{\Psi}\left[1+\frac{\left(\chi_{1}-\chi_{2}\right)(1-\hat{\tau}-\alpha)^{2} \hat{\Psi}_{\tau}}{\chi_{2}\left[(1-\hat{\tau}-\alpha) \hat{\Psi}_{\tau}-\hat{\Psi}\right]}\right] \\
\Omega(\Gamma) & =\left(1-\frac{1}{\alpha}\right)\left[\hat{x}_{2} \hat{\Psi}_{\tau} \hat{\Xi}+\hat{x}_{1} \hat{\Psi}+\rho \hat{\Psi}\right]+\hat{x}_{1} \rho
\end{aligned}
$$


We will use the Routh's Theorem in our analysis (see Gantmacher (1960, Chapter $\mathrm{XV})$ ). In our particular case this theorem says that the number of roots of $\Gamma$ with positive real parts is equal to the number of variations of sign in the sequence: $-1, \operatorname{tr}(\Gamma),-\Omega(\Gamma)+$ $\frac{\operatorname{det}(\Gamma)}{\operatorname{tr}(\Gamma)}, \operatorname{det}(\Gamma)$. From now, this sequence will be called the Routh's Sequence.

Although it is not possible to know from an analitical point of view the sign for the third term in the sequence, we can claim the following sufficient condition for local indeterminacy.

Proposition 8 If $1+\frac{\left(\chi_{1}-\chi_{2}\right)(1-\hat{\tau}-\alpha)^{2} \hat{\Psi}_{\tau}}{\chi_{2}\left[(1-\hat{\tau}-\alpha) \hat{\Psi}_{\tau}-\hat{\Psi}\right]}>0$ then there exist local indeterminacy.

Proof. If $1+\frac{\left(\chi_{1}-\chi_{2}\right)(1-\hat{\tau}-\alpha)^{2} \hat{\Psi}_{\tau}}{\chi_{2}\left[(1-\hat{\tau}-\alpha) \hat{\Psi}_{\tau}-\hat{\Psi}\right]}>0$ then $\operatorname{det}(\Gamma)<0$. Hence, given the Routh's Sequence, there are only two variations of sign, whatever the sign of $-\Omega(\Gamma)+\frac{\operatorname{det}(\Gamma)}{\operatorname{tr}(\Gamma)}$ be. Consequently, there are two roots with positive real part and one root with negative real part. Since the three variables in the system (45) are jump, there exist local determinacy only if the three roots have positive real part. Hence, under the condition $1+\frac{\left(\chi_{1}-\chi_{2}\right)(1-\hat{\tau}-\alpha)^{2} \hat{\Psi}_{\tau}}{\chi_{2}\left[(1-\hat{\tau}-\alpha) \hat{\Psi}_{\tau}-\hat{\Psi}\right]}>0$, the BGP is locally indeterminate.

Note that indeterminacy could also arise if $1+\frac{\left(\chi_{1}-\chi_{2}\right)(1-\hat{\tau}-\alpha)^{2} \hat{\Psi}_{\tau}}{\chi_{2}\left[(1-\hat{\tau}-\alpha) \hat{\Psi}_{\tau}-\hat{\Psi}\right]}<0$ and $-\Omega(\Gamma)+$ $\frac{\operatorname{det}(\Gamma)}{\operatorname{tr}(\Gamma)}>0$. In this case, there are two roots with negative real part because only one variation of sign would exist in the Routh's Sequence.

The implication of local indeterminacy is that there will be multiple paths converging to a given steady state. Hence, indeterminacy guarantees existence of a continuum of sunspot stationary equilibria, i.e., stochastic rational expectations equilibria determined by perturbations unrelated to the uncertainty in economic fundamentals. The interest of sunspot equilibria is that they provide a theoretical justification for 'animal spirits' underlying economic instability. In our model, indeterminacy is able to explain why two countries with similar fundamentals (preferences, technology and initial capital stock) might display different pollution paths even if such economies choose the same public policies.

In this sense, the result of local indeterminacy is able to explain the lack of empirical regularity in the pollution experiences of different economies; for example, the difficulty to find a regularity with respect to the Kuznets curve, the results being dependent on the sample of countries and years studied. That is, the past pollution experience of a developed country does not guarantee that a developing country will follow the same path along the time although the initial conditions and the environmental policies are similar.

The numerical examples shown in the preceding subsection are also useful to illustrate different situations of local indeterminacy.

The first example, defined by the following parameter set, $\alpha=0.7, A=1, \rho=$ $-\log (0.95), \eta=4, \chi_{1}=0.8, \chi_{2}=0.1$, displays local and global determinacy. That is, there exists one single converging path towards the single steady state.

The second example $\left(\alpha=0.7, A=1, \rho=-\log (0.95), \eta=3.310725, \chi_{1}=0.4\right.$, $\left.\chi_{2}=0.2\right)$ also displays local and global determinacy. 
The two BGPs of the third example $(\alpha=0.7, A=0.7, \rho=-\log (0.95), \eta=1$, $\left.\chi_{1}=0.7, \chi_{2}=0.6\right)$ are locally indeterminate. That is, there exist a continuum of equilibria converging to each BGP.

It is also possible to find examples of global indeterminacy with one locally determinate BGP and one locally indeterminate BGP. The following set of parameters $\alpha=0.7, A=1$, $\rho=-\log (0.95), \eta=3.31, \chi_{1}=0.4, \chi_{2}=0.2$, yields two BGPs characterized by $\hat{\tau}_{1}=0.76$, $\hat{\phi}_{1}=0.42, \hat{\gamma}_{1}=0.067$ and $\hat{\tau}_{2}=0.78, \hat{\phi}_{2}=0.43, \hat{\gamma}_{2}=0.059$. The first BGP is locally determinate and the second locally indeterminate.

\section{Planner solution}

We now derive the central planner solution as a first-best reference point. By comparing the first-order conditions of the decentralized solution with the corresponding first-order conditions of the planner solution, we are able to determine the first-best tax rates and spending composition (pigouvian tax rate and spending composition). Such pigouvian tax rate and spending composition let the economy reach the efficient path as a competitive equilibrium, that is, they internalize the externalities. We will show below that there are structural parameter sets for which is not possible to find a tax rate so that the competitive equilibrium replicates the efficient path, i.e., the pigouvian tax rate does not exist.

The benevolent central planner chooses consumption, public abatement activities and public production services in order to maximize the lifetime utility of the representative household subject to the flow resource constraint of the economy and the pollution function:

$$
\underset{\{c, k, P, g, z\}}{\operatorname{Max}} \int_{0}^{\infty} e^{-\rho t}[\ln c-\eta \ln P] d t
$$

subject to

$$
\begin{aligned}
c+\dot{k}+g+z & =A g^{1-\alpha} k^{\alpha} \\
P & =\frac{k^{\chi_{1}}}{z^{\chi_{2}}} \\
& k_{0} \text { given. }
\end{aligned}
$$

The current-value Hamiltonian for this optimization problem is

$$
H=\ln c-\eta \chi_{1} \ln k+\eta \chi_{2} \ln z+\nu\left(A g^{1-\alpha} k^{\alpha}-c-g-z\right),
$$


where $\nu$ is the co-state variable. The first-order conditions are given by

$$
\begin{aligned}
\frac{\partial H}{\partial c} & =0 \rightarrow \frac{1}{c}=\nu \\
\frac{\partial H}{\partial k} & =-\rho \nu+\dot{\nu} \rightarrow-\frac{\dot{\nu}}{\nu}=-\eta \chi_{1} \frac{1}{k \nu}+\alpha A g^{1-\alpha} k^{\alpha-1}-\rho \\
\frac{\partial H}{\partial g} & =0 \rightarrow \nu\left[(1-\alpha) A g^{-\alpha} k^{\alpha}-1\right]=0 \\
& \rightarrow \frac{k}{g}=\left[\frac{1}{(1-\alpha) A}\right]^{1 / \alpha}, \\
\frac{\partial H}{\partial z} & =0 \rightarrow \nu=\eta \chi_{2} \frac{1}{z} .
\end{aligned}
$$

The consumption to public abatement activities ratio is obtained from (46) and (49)

$$
\frac{c}{z}=\frac{1}{\eta \chi_{2}}
$$

which is a constant for all $t$, and the capital to public productive services ratio is also constant (48).

After eliminating the co-state variable by using equations (46), (47) and (48), the standard Euler condition for the central planner solution can be derived:

$$
\frac{\dot{c}}{c}=-\eta \chi_{1} \frac{c}{k}+A^{1 / \alpha} \alpha(1-\alpha)^{(1-\alpha) / \alpha}-\rho .
$$

Using the resources constraint, and the equations (48) and (50) we can obtain

$$
\frac{\dot{k}}{k}=A^{1 / \alpha}(1-\alpha)^{(1-\alpha) / \alpha}-\frac{c}{k}\left(1+\eta \chi_{2}\right)-[A(1-\alpha)]^{1 / \alpha}
$$

Proposition 9 This economy lacks transitional dynamics.

Proof. Let $x$ be the consumption to capital ratio $\left(\frac{c}{k}\right)$. The variation rate of $x$ is

$$
\frac{\dot{x}}{x}=\frac{\dot{c}}{c}-\frac{\dot{k}}{k} .
$$

From (51) and (52) we obtain

$$
\dot{x}=\left[1-\eta\left(\chi_{1}-\chi_{2}\right)\right] x^{2}-\rho x .
$$

Along a balanced growth path consumption and capital will grow at the same constant rate and $x$ will be constant. Hence, from (53), the steady state for $x$ is

$$
x^{*}=\frac{\rho}{\left[1-\eta\left(\chi_{1}-\chi_{2}\right)\right]} .
$$

Note that $x^{*}>0$ if and only if $1>\eta\left(\chi_{1}-\chi_{2}\right)$. 
The solution for differential equation (53) is

$$
x(t)=\frac{\rho}{\left[1-\eta\left(\chi_{1}-\chi_{2}\right)\right]-\left[\left[1-\eta\left(\chi_{1}-\chi_{2}\right)\right]-\frac{\rho}{x(0)}\right] e^{\rho t}} .
$$

From (55), one can obtain that only if $x=x^{*}, \forall t$, then $\lim _{t \rightarrow \infty} x(t)=x^{*}$. If $x(0) \neq x^{*}$, then $\lim _{t \rightarrow \infty} x(t)=0$, that is, the economy vanishes with the time. Hence, the consumption to capital ratio is always placed on the BGP, and so it is the capital to public productive services ratio (see (48)) and the consumption to public abatement activities ratio (see $(50))$.

Therefore, the command economy is globally and locally determinate since given $k(0)$, we obtain $c(0)=x^{*} k(0), z(0)=\eta \chi_{2} x^{*} k(0), g(0)=[(1-\alpha) A]^{1 / \alpha} k(0),{ }^{8}$ and

$$
q(t)=e^{\gamma_{P} t} q(0), \quad q=k, c, z, g,
$$

where $\gamma_{P}$ is the first-best growth rate, which can be obtained from (51) and (54):

$$
\gamma_{P}=-\eta \chi_{1} \frac{\rho}{\left[1-\eta\left(\chi_{1}-\chi_{2}\right)\right]}+A^{1 / \alpha} \alpha(1-\alpha)^{(1-\alpha) / \alpha}-\rho .
$$

Furthermore, the second-best economy can be defined for $\eta\left(\chi_{1}-\chi_{2}\right)<1$ or $\eta\left(\chi_{1}-\right.$ $\left.\chi_{2}\right)>1$, whilst the command economy is only defined for $\eta\left(\chi_{1}-\chi_{2}\right)<1$.

Now we address the design of the environmental policy that allows the economy reach the efficient path as a market equilibrium. Thus, we ilustrate how pigouvian tax and spending composition, if they exist, can be used to promote efficiency. However, under certain parameter sets, finding the first-best tax rate is not possible. In these cases, the implementation of the Ramsey-plan can be justifiable.

Therefore, we compute the pair $\left(\tau_{P}, \phi_{P}\right)$ such that $\gamma_{C}=\gamma_{P}$ and $\left(\frac{z}{g}\right)_{C}=\left(\frac{z}{g}\right)_{P}$, where $\gamma_{C}$ is given by (13), using (54), (50), and (48), the first-best $z$ to $g$ ratio is given by

$$
\left(\frac{z}{g}\right)_{P}=\frac{\eta \chi_{2} \rho}{[(1-\alpha) A]^{1 / \alpha}\left[1-\eta\left(\chi_{1}-\chi_{2}\right)\right]}
$$

and $\left(\frac{z}{g}\right)_{C}$ is obtained from $(10)$.

From the condition $\left(\frac{z}{g}\right)_{C}=\left(\frac{z}{g}\right)_{P}$, the pigouvian spending composition can be obtained:

$$
\phi_{P}=\frac{1}{1+\frac{[(1-\alpha) A]^{1 / \alpha}\left[1-\eta\left(\chi_{1}-\chi_{2}\right)\right]}{\eta \chi_{2} \rho}} .
$$

Whatever the structural parameter set, finding a pigouvian spending composition is always possible, since $\phi_{P}$ always falls between zero and one.

\footnotetext{
${ }^{8}$ Note that if transitional dynamic would exist, there would be a continuum of converging paths towards the BGP, that is, for each $\mathrm{x}(0)$ (or $\mathrm{c}(0)$, since $\mathrm{k}(0)$ is given) there exists a equilibrium path. Such continuum of equilibria could be indexed by $\mathrm{x}(0)$. In this case, the model would display local indeterminacy.
} 
Using (57) in the condition $\gamma_{C}=\gamma_{P}$, the pigouvian tax rate can be obtained from

$$
\frac{\gamma_{P}+\rho}{\alpha A^{1 / \alpha}\left(1-\phi_{P}\right)^{(1-\alpha) / \alpha}} \tau_{P}^{(\alpha-1) / \alpha}=1-\tau_{P} .
$$

The RHS of (58) is linear and decreasing in $\tau_{P} \in(0,1)$, with domain $(0,1)$. On the other hand, the $L H S$ of (58) is monotonically decreasing and convex in $\tau_{P} \in(0,1)$, with domain $\left(\frac{\gamma_{P}+\rho}{\alpha A^{1 / \alpha}\left(1-\phi_{P}\right)^{(1-\alpha) / \alpha}}, \infty\right)$. As a consequence, there can be two solutions, one solution or no solution for $\tau_{P}$. Hence, although the first-best allocations are globally determinate, the first-best tax policy can be indeterminate.

The examples used in the previous sections are useful to study the pigouvian tax rate and spending composition. However, only those examples verifying $1>\eta\left(\chi_{1}-\chi_{2}\right)$ are valid in the first-best framework.

The parameter set given by $\alpha=0.7, A=0.7, \rho=-\log (0.95), \eta=1, \chi_{1}=0.7$, $\chi_{2}=0.6$ yields the following second-best ${ }^{9}$ and first-best solutions:

\begin{tabular}{|c||c|c|}
\hline & second-best & first-best \\
\hline \hline$\gamma$ & $1.45 \%$ & \multirow{2}{*}{$16 \%$} \\
\hline \multirow{2}{*}{$\phi$} & $430 \%$ & \\
& $34 \%$ & $24 \%$ \\
\hline$\tau$ & $78 \%$ & \multirow{2}{*}{ no solution } \\
\hline
\end{tabular}

Note that the environmental quality is worse under the planner solution because i) pollution is growing at a larger rate, and ii) the share of public spending devoted to abatement is lower.

In this case, finding a pigouvian tax for which the economy reach the efficient path as a competitive market is not possible.

The parameter set given by $\alpha=0.7, A=1, \rho=-\log (0.95), \eta=3.31, \chi_{1}=0.4$, $\chi_{2}=0.2$ yields the following second-best and first-best solutions:

\begin{tabular}{|c||c|c|}
\hline & second-best & first-best \\
\hline \hline \multirow{2}{*}{$\gamma$} & $5.9 \%$ & \multirow{2}{*}{$16.6 \%$} \\
& $6.7 \%$ & \\
\hline \multirow{2}{*}{$\phi$} & $43 \%$ & \multirow{2}{*}{$36 \%$} \\
& $42 \%$ & \\
\hline \multirow{2}{*}{$\tau$} & $78 \%$ & $15 \%$ \\
& $76 \%$ & $50 \%$ \\
\hline
\end{tabular}

In this case the environmental quality is also worse under the planner solution for the same reasons cited above. Note that for this structural parameter set there exist two pigouvian taxes.

\section{Conclusion}

In a second-best framework, we study the dynamic properties of a general equilibrium model of endogenous growth with environmental externalities. In particular, we inves-

\footnotetext{
${ }^{9}$ Note that there are two BGP for the Ramsey problem for this parameter set.
} 
tigate the conditions for global and local indeterminacy, and their implications for the governmental control of pollution.

The modelled economy is characterized by: a) we assume that the capital stock used in production causes a negative environmental externality as a side product and this externality affects individuals' utility; aggregate pollution is a public bad which can be reduced by means of public abatement activities; b) the government expenditures are devoted to public abatement activities and to public productive services and these expenditures are financed by a pollution tax that the firms must pay; c) the government is benevolent and chooses its tax policy and composition of public spending by taking into account the decentralized competitive equilibrium.

We show that when we include pollution entering in the utility function together with the abatement public activities, the introduction of endogenously chosen economic policy can generate global and local indeterminacy, that is, there can be two steady states (global indeterminacy) both of which can be locally indeterminate (that is, there exist multiple converging paths to each state). On the contrary, the other type of externality in our economy (public production services), is not crucial to achieve the indeterminacy result, that is, if the public services do not provide any production externality, still is possible to obtain a global indeterminacy result.

Global and local indeterminacy are able to explain why two countries with similar fundamentals (preferences, technology and initial capital stock) might display different short and long run pollution paths regardless of the economic fundamentals.

Finally, we study the planner solution in order to derive the first-best tax rate by comparing the first order conditions of the competitive equilibrium with the first order conditions of the social planner solution. We find that there can be two, one or no pigouvian tax rate. When there exist two or one pigouvian tax rate, we show that the pigouvian tax is lower than the second-best tax. Furthermore, under the planner solution the environmental quality is worse since pollution is growing at a larger rate, and the share of public spending devoted to abatement is lower.

\section{References}

[1] Aiyagari, S.R.(1995), "The econometrics of indeterminacy: an applied study, a comment", Carnegie-Rochester Conf. Ser. Public Policy, 43, pp. 273-282.

[2] Barro, R. (1990), "Government spending in a simple model of economic growth", Journal of Political Economy, 98, pp. S103-S125.

[3] Benhabib, J. and R.E.A. Farmer (1994), "Indeterminacy and increasing returns", Journal of Economic Theory, 63, pp. 19-41.

[4] Benhabib, J. and R.E.A. Farmer (1996), "Indeterminacy and sector specific externalities", Journal of Monetary Economics 37, pp. 421-443.

[5] Benhabib, J. and Nishimura, K. (1998), "Indeterminacy and sunspots with constant returns", Journal of Economic Theory, 81, pp. 58-96. 
[6] Benhabib, J. and Perli, R. (1994), "Uniqueness and indeterminacy: transitional dynamics with multiple equilibria", Journal of Economic Theory, 63, pp. 113-142.

[7] Bennett, R.L. and R.E.A. Farmer (2000), "Indeterminacy with non-separable utility", Journal of Economic Theory 93, 118-143.

[8] Bovenberg, A. L. and R. A. de Mooij (1997), "Environmental tax reform and endogenous growth". Journal of Public Economics 63, pp. 207-237.

[9] de Bruyn, S.M. and R.J. Heintz (1999), "The environmental Kuznets curve hypothesis" in: J. van den Bergh, Handbook of Environmental and Resource Economics, Cheltenham: Edgar Elgar.

[10] Farmer, R.E.A. and J.T. Guo (1994), "Real business cycles and the animal spirits hypothesis, Journal of EconomicTheory, 63, pp. 42-73.

[11] Fernández, E., A. Novales and J. Ruiz (2003), "Indeterminacy under non-separability of public consumption and leisure in the utility function", Economic Modelling, 21, pp. $409-428$.

[12] Gantmacher, F.R. (1960), The Theory of Matrices, New York, Chelsea, vol. II.

[13] Ligthart, J. E. and F. van der Ploeg (1994), "Pollution, the cost of public funds and endogenous growth", Economics Letters, 46, pp. 351-361.

[14] Perli, R., (1998) "Indeterminacy, home production and the business cycle", Journal of Monetary Economics, 41, pp. 105-125.

[15] Mohtadi, H. (1996), "Environment, growth, and optimal policy design", Journal of Public Economics, 63, pp. 119-140.

[16] Park, H., and A. Philippopoulos (2004), "Indetermincy and fiscal policies in a growing economy", Journal of Economic Dynamics and Control, 28, pp. 645-660.

[17] Smulders, S. and R. Gradus (1996), "Pollution abatement and long-term growth", European Journal of Political Economy, 12, pp. 505-532.

[18] Weder, M. (1998) "Fickle consumers, durable goods and business cycles", Journal of Economic Theory, 81, pp. 37-57.

[19] Wen, Y., (1998), "Capacity utilization under increasing returns to scale", Journal of Economic Theory, 81, pp. 7-36.

[20] Xie, D. (1994), "Divergence in economic performance: transitional dynamics with multiple equilibria", Journal of Economic Theory, 63, pp. 97-112. 


\section{Appendix}

\subsection{Proof of proposition 5}

Given $\operatorname{LHS}(\hat{\tau})=\hat{\Psi}=(1-\hat{\tau}) \alpha A^{1 / \alpha}[(1-\alpha)(\hat{\tau}+\alpha)]^{(1-\alpha) / \alpha}$, first we show that it is decreasing for $\hat{\tau} \in[1-\alpha, 1)$.

Since

$$
\frac{\partial L H S(\hat{\tau})}{\partial \hat{\tau}}=A^{1 / \alpha}(1-\alpha)^{(1-\alpha) / \alpha}(\hat{\tau}+\alpha)^{(1-2 \alpha) / \alpha}\left[1-\alpha-\alpha^{2}-\hat{\tau}\right],
$$

then

$$
\frac{\partial L H S(\hat{\tau})}{\partial \hat{\tau}}<0, \text { for } \hat{\tau}>1-\alpha-\alpha^{2}
$$

Since $\hat{\tau}>1-\alpha>1-\alpha-\alpha^{2}$, then $\operatorname{LHS}(\hat{\tau})$ is decreasing for the range of $\hat{\tau}$.

Second, we show that $\operatorname{LHS}(\hat{\tau})$ is concave in $\hat{\tau}$.

$$
\frac{\partial^{2} L H S(\hat{\tau})}{\partial \hat{\tau}^{2}}=\frac{\hat{\Psi}}{[(1-\hat{\tau})(\hat{\tau}+\alpha) \alpha]^{2}}\left[\left(1-\alpha-\alpha^{2}-\hat{\tau}\right)(2 \hat{\tau}+\alpha) \alpha-(1-\hat{\tau})(\hat{\tau}+\alpha) \alpha\right]<0
$$

since $\hat{\tau}>1-\alpha-\alpha^{2}$, like we shown previously.

Furthermore: $\lim _{\hat{\tau} \rightarrow(1-\alpha)^{+}} \hat{\Psi}=\alpha^{2} A^{1 / \alpha}[(1-\alpha)]^{(1-\alpha) / \alpha}>0 ; \lim _{\hat{\tau} \rightarrow 1^{-}} \hat{\Psi}=0$. Therefore, the function is positive $\forall \tau \in[1-\alpha, 1)$.

\subsection{Proof of Proposition 6 and 7}

Define $\Delta=1-\eta\left(\chi_{1}-\chi_{2}\right)$ and $\Pi=\chi_{2} \frac{1+\alpha}{\alpha}-\chi_{1}$. When $\hat{\tau}>(1-\alpha), \operatorname{RHS}(\hat{\tau})=$ $\frac{\rho \eta}{\Delta}\left[\chi_{2} \frac{\hat{\tau}+\alpha}{\hat{\tau}+\alpha-1}-\chi_{1}\right]$ is continuous, and decreasing (increasing) in $\hat{\tau}$ if $\Delta>(<) 0$ :

$$
\begin{aligned}
\frac{\partial R H S(\hat{\tau})}{\partial \hat{\tau}} & =-\frac{\rho \eta}{\Delta} \frac{1}{[(\hat{\tau}+\alpha)-1]^{2}} \\
\frac{\partial^{2} R H S(\hat{\tau})}{\partial \hat{\tau}^{2}} & =\frac{2 \rho \eta}{\Delta} \frac{1}{[(\hat{\tau}+\alpha)-1]^{3}}
\end{aligned}
$$

and hence:

$$
\begin{aligned}
\text { i) If } \Delta & <0 \text { and } \Pi<0: \\
\frac{\partial R H S(\hat{\tau})}{\partial \hat{\tau}} & >0 ; \frac{\partial^{2} R H S(\hat{\tau})}{\partial \hat{\tau}^{2}}<0 ; \\
\lim _{\hat{\tau} \rightarrow(1-\alpha)^{+}} R H S(\hat{\tau}) & =-\infty ; \lim _{\hat{\tau} \rightarrow 1^{-}} R H S(\hat{\tau})=\kappa>0
\end{aligned}
$$


therefore, $R H S(\hat{\tau})$ is increasing and concave, with domain $(-\infty, \kappa)$.

$$
\begin{aligned}
\text { ii) If } \Delta & <0 \text { and } \Pi>0: \\
\frac{\partial R H S(\hat{\tau})}{\partial \hat{\tau}} & >0 ; \frac{\partial^{2} R H S(\hat{\tau})}{\partial \hat{\tau}^{2}}<0 ; \\
\lim _{\hat{\tau} \rightarrow(1-\alpha)^{+}} R H S(\hat{\tau}) & =-\infty ; \lim _{\hat{\tau} \rightarrow 1^{-}} R H S(\hat{\tau})=\kappa<0
\end{aligned}
$$

therefore, $\operatorname{RHS}(\hat{\tau})$ is increasing and concave, with domain $(-\infty, \kappa)$.

$$
\begin{aligned}
\text { iii) If } \Delta & >0 \text { and } \Pi<0: \\
\frac{\partial R H S(\hat{\tau})}{\partial \hat{\tau}} & <0 ; \frac{\partial^{2} R H S(\hat{\tau})}{\partial \hat{\tau}^{2}}>0 ; \\
\lim _{\hat{\tau} \rightarrow(1-\alpha)^{+}} R H S(\hat{\tau}) & =\infty ; \lim _{\hat{\tau} \rightarrow 1^{-}} R H S(\hat{\tau})=\kappa<0
\end{aligned}
$$

therefore, $R H S(\hat{\tau})$ is decreasing and convex, with domain $(\kappa, \infty)$.

$$
\begin{aligned}
\text { iv) If } \Delta & >0 \text { and } \Pi>0: \\
\frac{\partial R H S(\hat{\tau})}{\partial \hat{\tau}} & <0 ; \frac{\partial^{2} R H S(\hat{\tau})}{\partial \hat{\tau}^{2}}>0 ; \\
\lim _{\hat{\tau} \rightarrow(1-\alpha)^{+}} R H S(\hat{\tau}) & =\infty ; \lim _{\hat{\tau} \rightarrow 1^{-}} R H S(\hat{\tau})=\kappa>0
\end{aligned}
$$

therefore, $R H S(\hat{\tau})$ is decreasing and convex, with domain $(\kappa, \infty)$. 


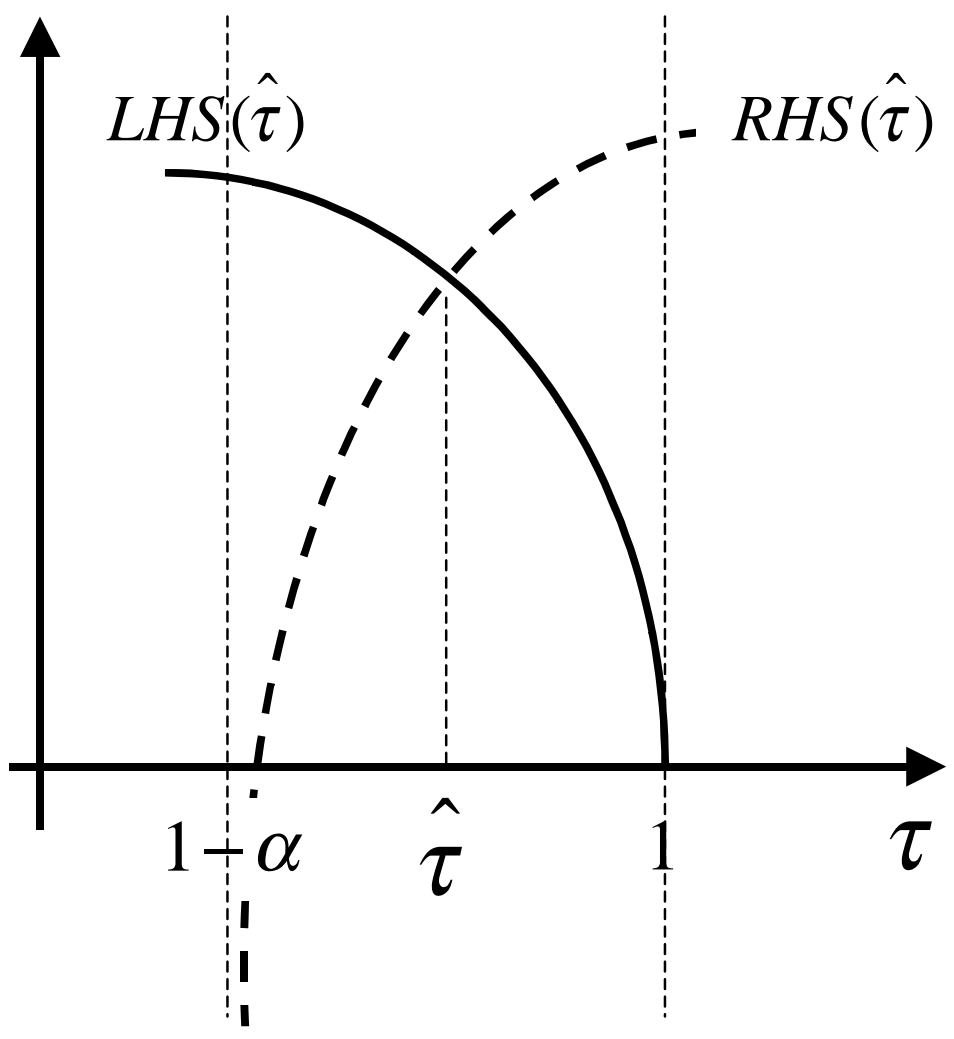

Figure 1a

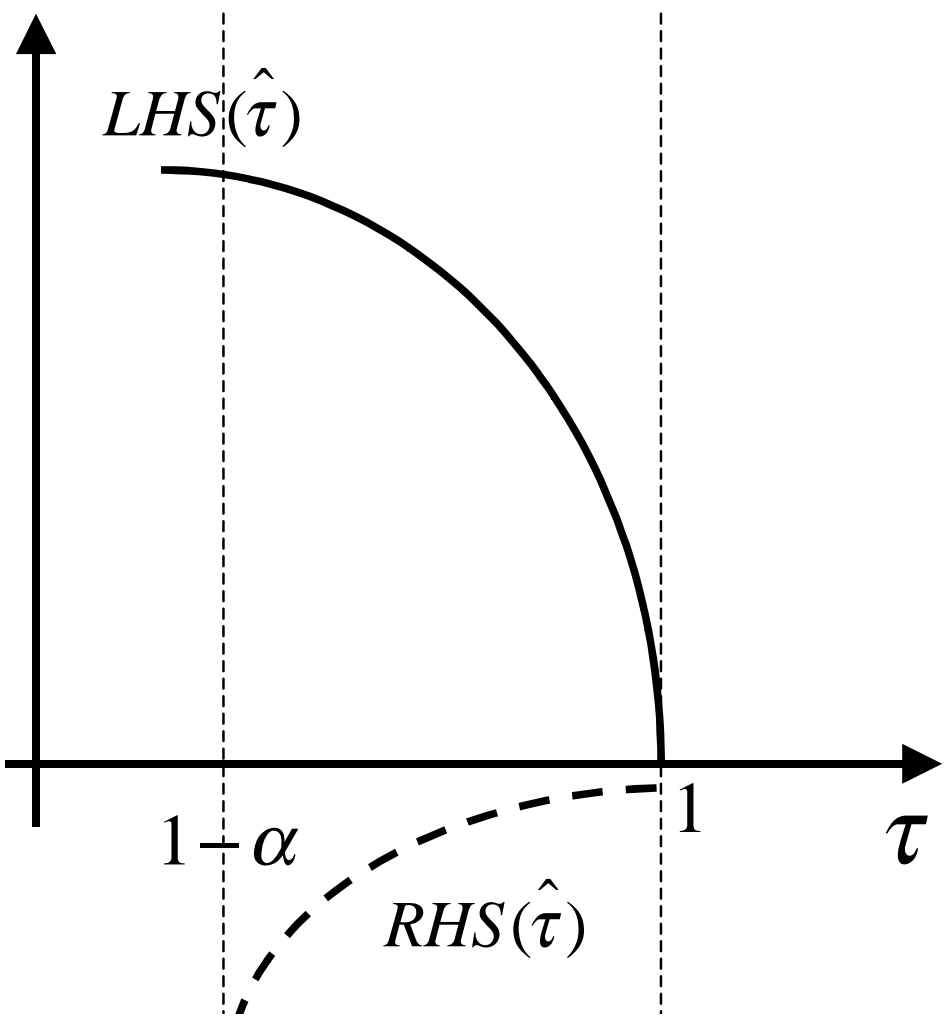

Figure 1b 


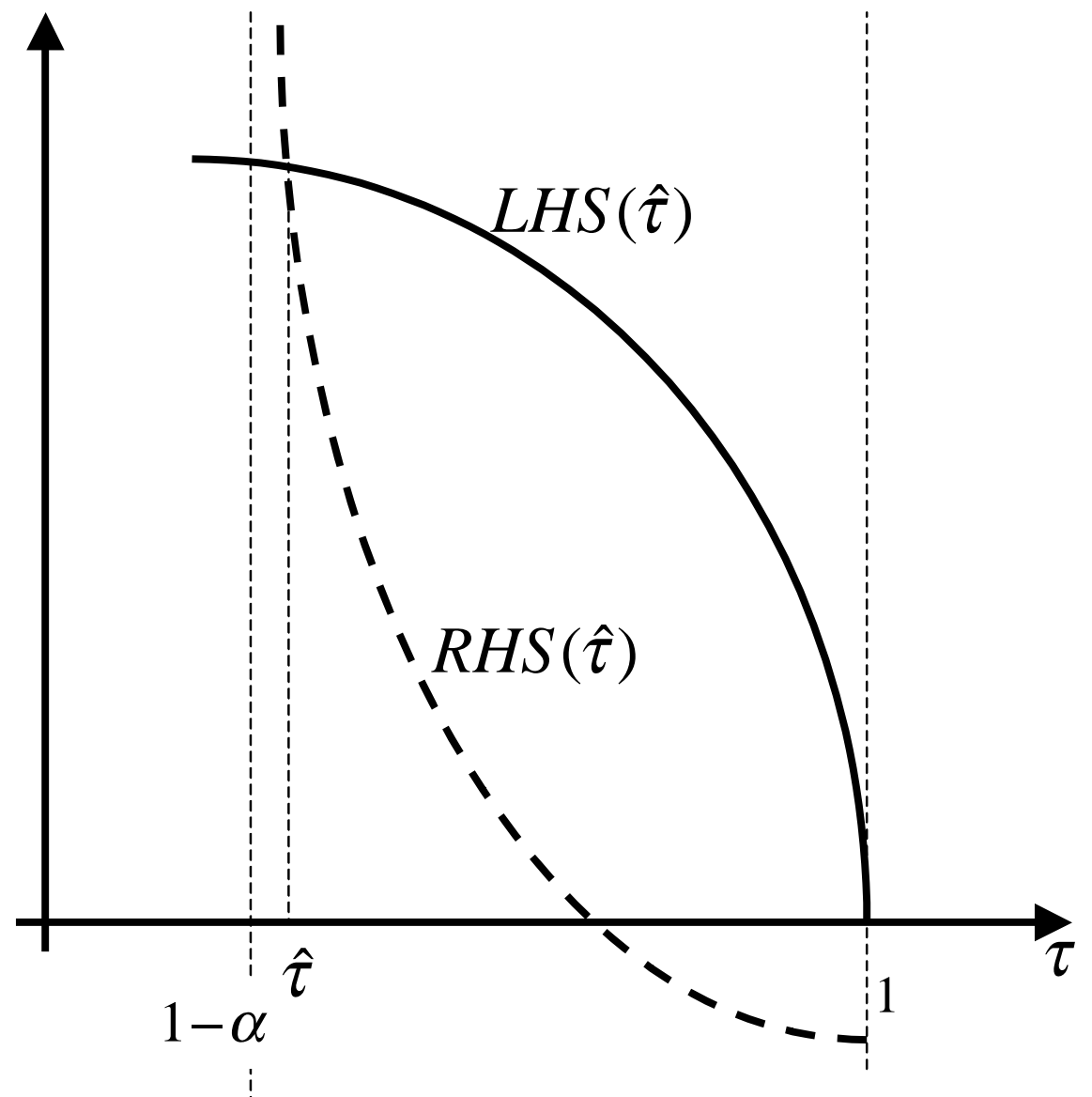

Figure 2 


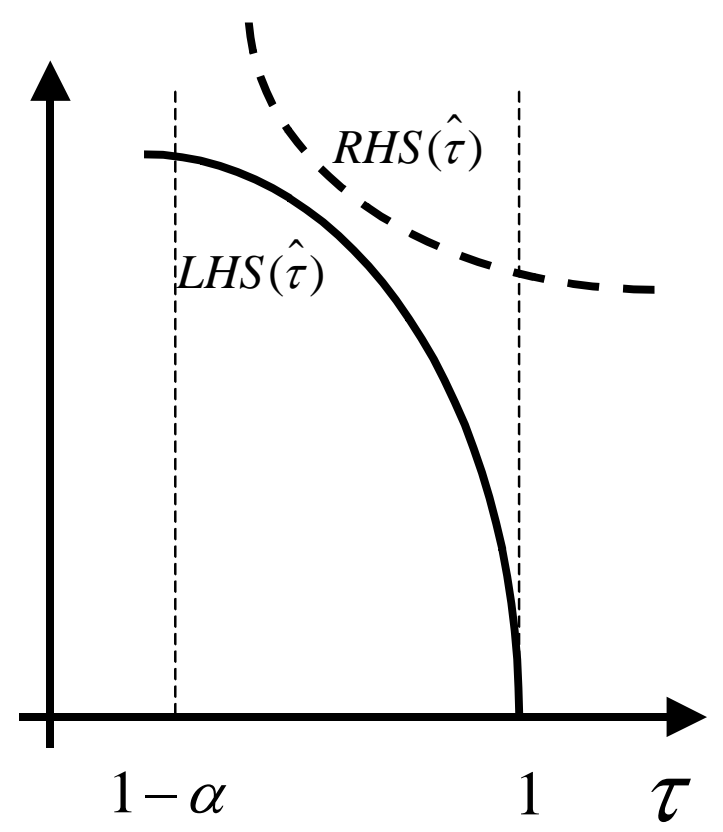

Figure 3a

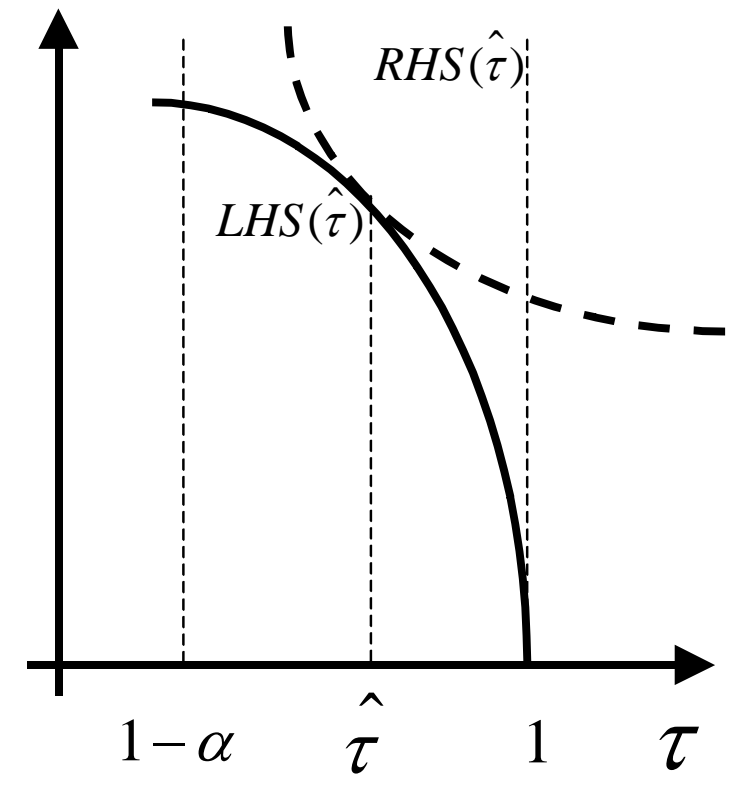

Figure 3b

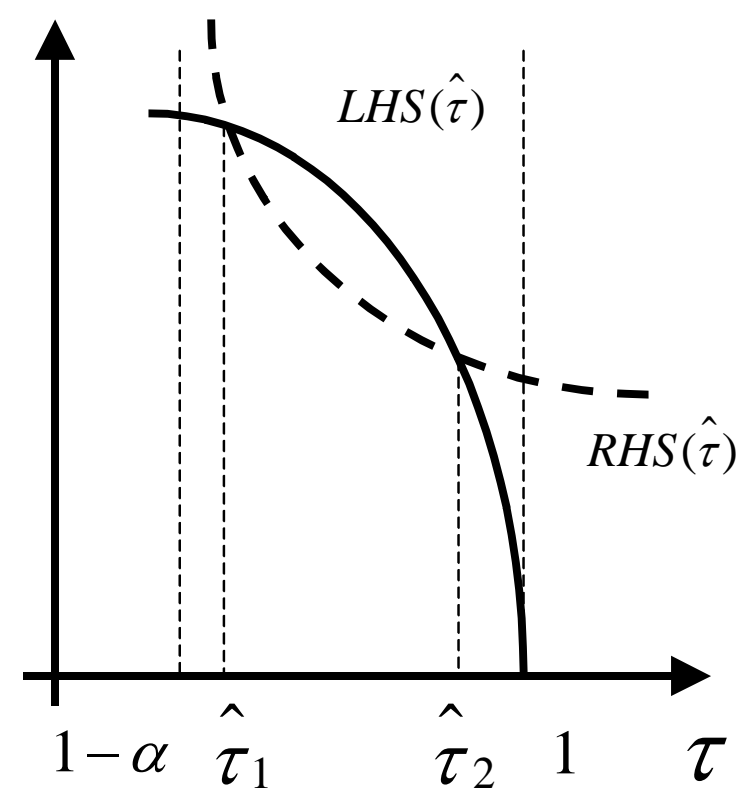

Figure 3c 